\title{
A Study of the Termination Reaction Rate of a Polymer Radical of Definite Degree of Polymerization. I. Polystyryl Radical
}

\author{
Takashi Fukutomi, Masakazu Toyoura, and Toshio KaKuraI \\ Tokyo Institute of Technology, 2-12, Ookayama, Meguro-ku, Tokyo 152, Japan.
}

(Received July 12, 1975)

\begin{abstract}
The difunctional polystyryl anion of a definite degree of polymerization was reacted with triphenylmethylbromide to form a polystyryl radical. The ratio $k_{i j} / k_{1}{ }^{2}$, where $k_{i j}$ is the rate constant of the termination reaction between two polymer radicals (with $i$ and $j$ as the degree of polymerization), and $k_{1}$ is the rate constant of the termination reaction between a polymer radical and the radical deactivators, was estimated by GPC (gel-permeation chromatography) analysis on the polymer produced from the free radical species formed by the anion-radical transformation reaction. It was found that the ratio $k_{i j} / k_{1}{ }^{2}$ increases when the polymer concentration and the value $j-1(j=1,2,3)$ increase. On the contrary, when the polymer concentration decreases, $k_{i j} / k_{1}{ }^{2}$ decreases. The results were discussed from the viewpoint of a diffusion-controlled reaction.
\end{abstract}

KEY WORDS Radical / Termination / Definite Degree of Polymerization / Steady State / Gel-Permeation Chromatography /

Kinetic studies concerning the termination reaction of polymer radicals have been carried out by many authors since the so-called "geleffect" ${ }^{\prime 1,2}$ was discovered. In some of these papers, the value ${ }_{2} k_{\mathrm{t}}$ (rate constant for the termination reaction between two polymer radicals) was discussed in connection with the viscosity of the medium, the flexibility of polymer chains, the interaction between solvent and polymer segments, the degree of polymerization, etc.

The facts that ${ }_{2} k_{\mathrm{t}}$ is inversely proportional to the viscosity of the medium, ${ }^{3,4}$ and has some relation with the flexibility of a polymer chain, ${ }^{5,6}$ and with the nature of the solvents (solubility of polymer to solvent) $)^{3}$ have been reported previously. Concerning the effect of the degree of polymerization, some experimental results were obtained by Schulz and his coworker, ${ }^{7}$ and Borgwardt and his coworkers. 8 But the former used polydisperse polymers and the latter used a polymer on which the reactive groups were located at indifinite positions.

On the other hand, many authors have proposed various diffusion-controlled models for this reaction,,$^{9-17}$ by assuming an analogy between this reaction and the coaguration process, ${ }^{11-13}$ by considering the microviscosity when the polymer ends penetrate into the medium occupied by the other polymer molecule ${ }^{14}$ or by taking into account the relaxation phenomena and the excluded volume effect of the polymer molecules. ${ }^{15-17}$

Thus the dependency of ${ }_{2} k_{\mathrm{t}}$ on the degree of polymerization has been derived by several authors according to the above models, while it may be considered that the quantitative data needed to decide what kind of model is suitable to express the termination rate are not sufficient. Moreover, the influence of the polymer concentration is not clear, even qualitatively.

The difficulty of clarifying these points lies mainly in the absence of any method to deal with polymer radicals of a definite degree of polymerization.

In this study, we attempt to establish a method to measure the rate constant ${ }_{2} k_{\mathrm{t}}$ for polymer radicals of a definite degree of polymerization, using living polymer, transforming the styryl anion into its radical with triphenylmethylbromide 
(TPMB), and employing GPC (gel-permeation chromatography) to measure the molecular weight distribution.

As an actual problem, the absolute vale of ${ }_{2} k_{\mathrm{t}}$ is rather difficult to measure and, therefore, we tried to determine the value $k_{i j} / k_{1}^{2}$, where $k_{1}$ is the rate constant of a termination reaction between the polymer radical and the solvent or other radical deactivator.

\section{EXPERIMENTAL}

\section{Living Polystyrene}

Living polystyrene solution was prepared using $\alpha$-methyl styrene living tetramer (counter ion: $\mathrm{Na}^{+}$) as the initiator and tetrahydrofuran (THF) as the solvent at $-78^{\circ} \mathrm{C}$ under vacuum. After polymerization, the living polymer solution was divided into $6-7$ small ampoules $(v=3-5 \mathrm{ml})$,

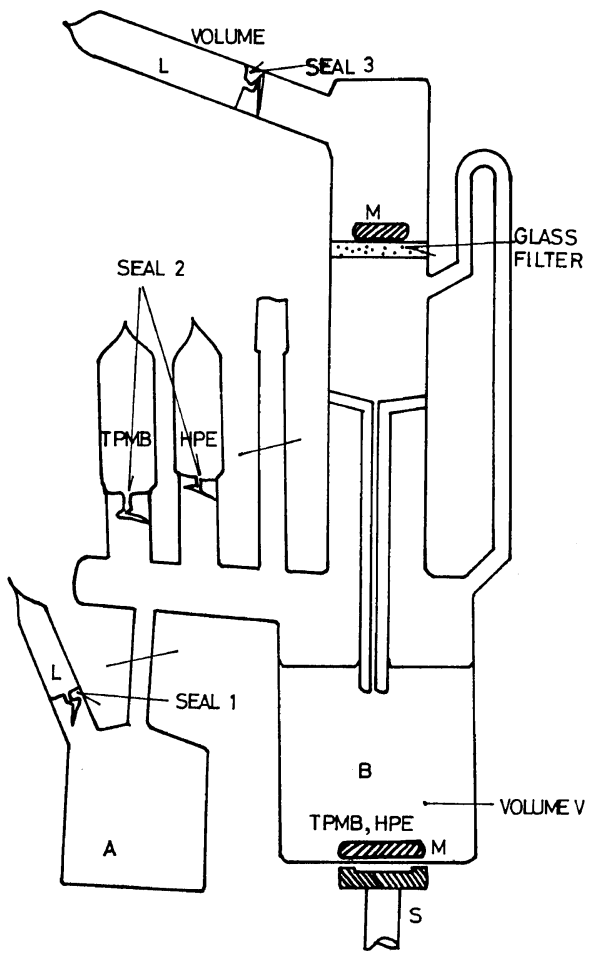

Figure 1. The apparatus to transform styryl anion to its radical and to achieve the steady state of the concentration of reactive species: L, THF solution of living polymer; TPMB, triphenylmethylbromide; HPE, hexaphenylethane; [TPMB]V, $[\mathrm{HPE}] \mathrm{V} \gg[\mathrm{L}] \cup \mathrm{M}$; magnet coated with glass; $\mathrm{s}$, stirrer. and one of them was used to determine the concentration of the living ends by deactivation with $n$-butyl bromide. Another one was used to determine the degree of polymerization and the polymer concentration by deactivation with methanol.

The degree of polymerization was determined by viscometry, using the following relationship: ${ }^{18}$

$$
\bar{M}_{V}=1.7 \times 10^{5}[\eta]^{1.35} \quad\left(30^{\circ} \mathrm{C} \text {, in toluene }\right)
$$

Preparation of Hexaphenylethane (HPE) (or Dimer of Triphenyl Methyl Radical ${ }^{22}$

HPE was prepared by the method described by Preckel and Selwood. ${ }^{19}$ The silver powder and TPMB were reacted in the apparatus shown in Figure 2 at room temperature for 3-4 days in benzene. When the reaction was over, the solution was filtered and transferred to part B. Then the benzene was evaporated to part A by cooling A. The conversion of TPMB was calculated from the increment caused by the formation of $\mathrm{AgBr}$.

The HPE thus prepared was then dissolved in THF under vacuum, and divided into small, graduated ampoules.

\section{Deactivation of Styryl Anion with TPMB}

The apparatus shown in Figure 1 was used to deactivate the polystyryl anion with TPMB in order to form the polystyryl radical and to

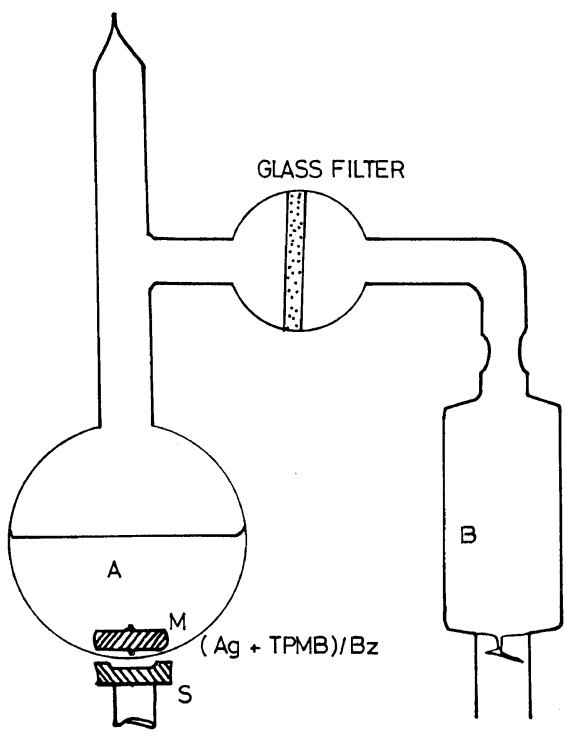

Figure 2. The apparatus to synthetize hexaphenylethane from triphenylmethylbromide in vacuo. 
continue the subsequent radical reactions.

At first, the breakable seal 1 was broken, and all parts of the apparatus were washed by the solution; then this solution was completely brought into part $\mathrm{A}$, and pure THF only was condensed by cooling part $\mathrm{B}$ to $-78^{\circ} \mathrm{C}$. After sealing off bottle $\mathrm{A}$, seal 2 was broken. Then the apparatus was immersed totally in the water bath $\left(30^{\circ} \mathrm{C}\right)$, seal 3 was broken, and the living polymer solution was introduced slowly and continuously into part B through the glass filter and the capillary.

The time $\tau$ for the living polymer solution to flow out completely was measured.

Other reaction conditions were as follows:

$\left.V \gg v ; \quad V[\mathrm{TPMB}], V[\mathrm{HPE}] \gg{ }^{-} \mathrm{P}_{1}^{-}\right]$

$\tau ; 10^{3}-10^{2} \mathrm{sec}$ (very long time compared to the life time of the polymer radical and anion)

\section{GPC and its Analysis}

The experimental conditions were as follows:

Instrument Waters Instrument, Type 200 Temp $\quad 20^{\circ} \mathrm{C}$

Polymer concn $20-30 \mathrm{mg} / 10 \mathrm{ml} \mathrm{THF}$

Solvent THF

Injection time $90 \mathrm{sec}$

3 Columns for sample $10^{7}, 10^{6}, 10^{5} \AA$ porosity

3 Columns for reference $10^{4}, 10^{3}, 10^{2} \AA$ porosity

The weight fraction of each polymer composition was calculated by using Tung's expression: ${ }^{20}$

$$
W(v)=\int\left(\frac{c}{\pi}\right)^{1 / 2} h_{j}\left(v_{j}\right) \exp \left[-c\left(v_{j}-v\right)^{2}\right] \mathrm{d} v_{j}
$$

where $W(v)$ means the observed GPC, $v$ is the elution volume, and $v_{j}$ means the elution volume where GPC shows a maximum peak when $j$-mer

Table I. Reaction conditions and results (I)

\begin{tabular}{crcccccc}
\hline Expt no. & $\begin{array}{c}t, \\
\mathrm{sec}\end{array}$ & $\begin{array}{c}\text { Polymer concn, }{ }^{\mathrm{a}} \\
\mathrm{g} / 100 \mathrm{~m} l\end{array}$ & $\begin{array}{c}2 \gamma,{ }^{\mathrm{b}} \\
\mathrm{mol} / l \mathrm{sec}\end{array}$ & $\begin{array}{c}{\left[\mathrm{P}_{1}\right] \times 10^{4},} \\
\mathrm{~mol} / l\end{array}$ & $\begin{array}{c}{\left[\mathrm{P}_{2}\right] \times 10^{4},} \\
\mathrm{~mol} / l\end{array}$ & $\begin{array}{c}{\left[\mathrm{P}_{3}\right] \times 10^{4},} \\
\mathrm{~mol} / l\end{array}$ & $\begin{array}{c}k_{11} / k_{1}{ }^{2}, \\
l \mathrm{sec} / \mathrm{mol}\end{array}$ \\
\hline 1 & 1739.2 & 2.13 & $8.9 \times 10^{-7}$ & 4.22 & 1.53 & 0.61 & $2.7 \times 10^{5}$ \\
2 & 227.4 & 1.21 & $4.1 \times 10^{-6}$ & 2.79 & 0.89 & 0.00 & $0.5 \times 10^{5}$ \\
3 & 92.7 & 0.63 & $5.2 \times 10^{-6}$ & 1.78 & 0.32 & 0.00 & $0.2 \times 10^{5}$ \\
4 & 1009.8 & 1.27 & $4.5 \times 10^{-7}$ & 1.75 & 0.25 & 0.00 & $2.1 \times 10^{5}$ \\
5 & 886.7 & 1.06 & $3.9 \times 10^{-7}$ & 1.38 & 0.18 & 0.00 & $2.2 \times 10^{5}$ \\
6 & 216.7 & 1.03 & $1.6 \times 10^{-6}$ & 1.22 & 0.25 & 0.00 & $2.2 \times 10^{5}$ \\
7 & 495.5 & 0.67 & $5.8 \times 10^{-7}$ & 1.09 & 0.17 & 0.00 & $1.8 \times 10^{5}$ \\
8 & 561.1 & 0.37 & $2.4 \times 10^{-7}$ & 0.60 & 0.04 & 0.00 & $1.9 \times 10^{5}$ \\
\hline
\end{tabular}

a At the final state of the reaction.

b This value is equal to the inflow velocity of living ends, and contains ca. 10-20\% error caused by the local condensation of THF when the solution was divided into small ampoules, in some cases.

Other reaction conditions

Expt no. $1-3: \bar{M}_{V}$ of living polymer, $1.9 \times 10^{4}$ $V+v, 49.2 \pm 3.6 \mathrm{ml}, v<4.57 \mathrm{ml}$

Expt no. 4-8: $\bar{M}_{V}$ of living polymer, $5.1 \times 10^{4}$; [TPMB], $1.11 \times 10^{-2}(\mathrm{~mol} / \mathrm{l})$; $V+v, 49.5 \pm 4.1 \mathrm{ml}, v<4.58 \mathrm{ml}$; [HPE], $\quad 5.43 \times 10^{-5}(\mathrm{~mol} / l)$. [TPMB], $0.941 \times 10^{-2}(\mathrm{~mol} / \mathrm{l})$; [HPE], $5.56 \times 10^{-5}(\mathrm{~mol} / l)$.

Table II. Reaction conditions and results (II) ${ }^{\mathrm{a}}$

\begin{tabular}{|c|c|c|c|c|c|c|c|c|c|c|}
\hline $\begin{array}{l}\text { Expt } \\
\text { no. }\end{array}$ & $\begin{array}{c}t \\
\mathrm{sec}\end{array}$ & $\begin{array}{l}\text { Polymer } \\
\text { concn, } \\
\mathrm{g} / 100 \mathrm{~m} l\end{array}$ & $\begin{array}{c}2 \gamma, \\
\mathrm{mol} / l \mathrm{sec}\end{array}$ & $\underset{\mathrm{mol} / \mathrm{l}}{\left[\mathrm{P}_{1}\right] \times 10^{4}}$ & $\begin{array}{c}{\left[\mathrm{P}_{2}\right] \times 10^{4},} \\
\mathrm{~mol} / \mathrm{l}\end{array}$ & $\begin{array}{c}{\left[\mathrm{P}_{3}\right] \times 10^{4},} \\
\mathrm{~mol} / \mathrm{l}\end{array}$ & $\begin{array}{c}{\left[\mathrm{P}_{4}\right] \times 10^{4},} \\
\mathrm{~mol} / \mathrm{l}\end{array}$ & $\begin{array}{c}k_{11} / k_{1}^{2} \\
l \mathrm{sec} / \mathrm{mol}\end{array}$ & $\begin{array}{c}k_{12} / k_{1}^{2} \\
l \mathrm{sec} / \mathrm{mol}\end{array}$ & $\begin{array}{c}k_{13} / k_{1}^{2} \\
l \mathrm{sec} / \mathrm{mol}\end{array}$ \\
\hline 1 & 400.8 & 2.50 & $9.10 \times 10^{-6}$ & 8.00 & 1.68 & 0.44 & 1.39 & $1.5 \times 10^{-4}$ & $3.5 \times 10^{-4}$ & $120 \times 10^{-4}$ \\
\hline 2 & 379.0 & 2.20 & $8.46 \times 10^{-6}$ & 7.71 & 1.13 & 0.50 & 1.14 & $1.2 \times 10^{-4}$ & $6.3 \times 10^{-4}$ & $90 \times 10^{-4}$ \\
\hline 3 & 41.2 & 0.67 & $23.1 \times 10^{-6}$ & 2.83 & 0.24 & 0.12 & 0.27 & $0.25 \times 10^{-4}$ & $2.6 \times 10^{-4}$ & $32 \times 10^{-4}$ \\
\hline
\end{tabular}

a Other reaction conditions:

$\bar{M}_{V}$ of living polymer, $1.4 \times 10^{4} ; V+v, 49.3 \pm 1.5 \mathrm{ml} ; v<4.58 \mathrm{ml} ;$ [TPMB], $0.983 \times 10^{-2}(\mathrm{~mol} / l) ;[\mathrm{HPE}]$, $5.80 \times 10^{-4}(\mathrm{~mol} / l)$. 
only is measured.

The reaction conditions and the observed values of $\left[\mathrm{P}_{1}\right],\left[\mathrm{P}_{2}\right],\left[\mathrm{P}_{3}\right]$, and $\left[\mathrm{P}_{4}\right]$ are reported in Table I and II. In both experimental set, the concentration of living ends is in the order of $10^{-2}-10^{-3}(\mathrm{~mol} / l)$.

Method for the Determination of the Ratios of the Rate Constants

As Richards, et al. ${ }^{21}$ have reported previously polystyryl anion can be transformed to polystyryl radical by TPMB

(polystyrene) $^{-} \mathrm{Me}^{+}+\mathrm{TPMB}$

$$
\stackrel{k_{\mathrm{T}}}{\longrightarrow} \text { (polystyrene). }+. \mathrm{C} \phi_{3}
$$

then the polystyryl radical continue the following reactions

2 (polystyrene).

$\stackrel{{ }^{2 k_{\mathrm{t}}}}{\longrightarrow}$ (polystyrene)-(polystyrene)

(polystyrene)..$+ \mathrm{C} \phi_{3} \stackrel{k^{\prime}}{\longrightarrow}$ (polystyrene)-C $\phi_{3}$

(polystyrene). $+\mathrm{BrC} \phi_{3}$

$$
\stackrel{k^{\prime \prime}}{\longrightarrow} \text { (polystyrene)- } \mathrm{Br}+. \mathrm{C} \phi_{3}
$$

(polystyrene). + solvent

$$
\stackrel{k^{\prime \prime \prime} \prime}{\longrightarrow} \text { (polystyrene)- } \mathrm{H}+. \mathrm{R}
$$

If the difunctional polymer is used, the kinetic expressions are as follows.

$$
\begin{aligned}
& \frac{\mathrm{d}\left[{ }^{-} \mathbf{P}_{j}^{-}\right]}{\mathrm{d} t}=\sum_{l+m=j} k_{l m}\left[{ }^{-} \mathbf{P}_{i}\right]\left[{ }^{-} \mathbf{P}_{m}^{\cdot}\right]-2 k_{\mathrm{D}}\left[{ }^{-} \mathbf{P}_{j}^{-}\right] \\
& \frac{\mathrm{d}\left[{ }^{-} \mathrm{P}_{j}\right]}{\mathrm{d} t}=2 \sum_{l+m=j} k_{l m}\left[{ }^{-} \mathrm{P}_{i}\right]\left[\mathrm{P}_{m}\right]+2 k_{\mathrm{D}}\left[{ }^{-} \mathrm{P}_{j}^{-}\right] \\
& \left.-\sum_{i=1} k_{i j}\left[{ }^{-} \mathbf{P}_{i}^{\cdot}\right]+2\left[\mathbf{P}_{i}^{\cdot}\right]+\left[\mathbf{P}_{i}\right]\right)\left[{ }^{-} \mathbf{P}_{j}\right] \\
& -k_{1}\left[{ }^{-} \mathbf{P}_{j}\right]-k_{\mathrm{D}}\left[{ }^{-} \mathbf{P}_{j}\right] \\
& \frac{\mathrm{d}\left[\cdot \mathbf{P}_{j}\right]}{\mathrm{d} t}=4 \sum_{l+m=j} k_{l m}\left[\mathbf{P}_{i}\right]\left[\mathbf{P}_{m}\right]+k_{\mathrm{D}}\left[{ }^{-} \mathbf{P}_{j}\right] \\
& -2 \sum_{i=1} k_{i j}\left(\left[{ }^{-} \mathbf{P}_{i}^{\cdot}\right]+2\left[\mathbf{P}_{i}\right]+\left[\mathbf{P}_{i}\right]\right)\left[\mathbf{P}_{j}\right] \\
& -2 k_{1}\left[\mathrm{P}_{j}\right] \\
& \frac{\mathrm{d}\left[\mathbf{P}_{j}^{-}\right]}{\mathrm{d} t}=\sum_{l+m=j} k_{l m}\left[{ }^{-} \mathbf{P}_{i}\right]\left[\mathbf{P}_{m}^{\cdot}\right]+k_{1}\left[{ }^{-} \mathbf{P}_{j}^{j}\right]-k_{\mathrm{D}}\left[\mathbf{P}_{j}^{-}\right] \\
& \frac{\mathrm{d}\left[\mathrm{P}_{j}^{\prime}\right]}{\mathrm{d} t}=2 \sum_{l+m=j} k_{l m}\left[\mathrm{P}_{i}\right]\left[\mathrm{P}_{m}^{\cdot}\right]+2 k_{1}\left[\mathbf{P}_{j}^{j}\right]+k_{\mathrm{D}}\left[\mathrm{P}_{j}^{-}\right] \\
& -\sum_{i=1} k_{i j}\left(\left[{ }^{-} \mathbf{P}_{i}^{i}\right]+2\left[\mathbf{P}_{i}^{j}\right]+\left[\mathbf{P}_{i}^{i}\right]\right)\left[\mathbf{P}_{j}^{j}\right] \\
& -k_{1}\left[\mathrm{P}_{j}\right] \\
& \left(k_{\mathrm{D}}=k_{\mathrm{T}}[\mathrm{TPMB}]\right. \text {, } \\
& \left.\left.k_{1}=k^{\prime}\left[. \mathrm{C} \phi_{3}\right]+k^{\prime \prime}[\mathrm{TPMB}]+k^{\prime \prime \prime} \text { [solvent }\right]\right)
\end{aligned}
$$

Polymer J., Vol. 8, No. 2, 1976 where $\left[{ }^{-} \mathbf{P}_{j}^{-}\right],\left[{ }^{-} \mathbf{P}_{j}\right],\left[{ }^{[} \mathbf{P}_{j}\right],\left[\mathbf{P}_{j}^{-}\right],\left[\mathbf{P}_{j}\right]$, and $\left[\mathbf{P}_{j}\right]$ are the polymer concentrations when the two ends are both anions, one end is still anion and one end is transformed to radical, both ends are transformed to radicals, one end is anion and one end is inactive, one end is radical and one end is inactive, and both ends are inactive, respectively. The degree of polymerization is equal to the value $j \times$ (degree of polymerization of the living polymer).

If the apparatus shown in Figure 1 is used to transform the living polystyrene to its radical, we may consider that the steady-state with respect to all the active species and the constancies of $k_{1}$ and $k_{\mathrm{D}}$ are achieved.

In the actual case of the present study, the amounts of $P_{2}, P_{3}$, and $P_{4}$ were very small compared to the amount of $P_{1}$; this fact means that the reaction between active species with suffix $j$ higher than 2 is rather improbable. So we may consider that $\mathbf{P}_{j+1}$ is produced by the reaction between $\mathrm{P}_{j}$ and $\mathrm{P}_{i}$. Moreover, on polymers with a degree of polymerization higher than $4 \times$ (degree of polymerization of living polymer) were observed in this study.

Then the kinetic expressions are as follows, considering the above conditions and the steadystate approximation:

$$
\begin{aligned}
& \frac{\mathrm{d}\left[{ }^{-} \mathrm{P}_{1}^{-}\right]}{\mathrm{d} t}=\frac{\mathrm{d} v}{\mathrm{~d} t} \frac{C}{V}-2 k_{\mathrm{D}}\left[-\mathrm{P}_{1}^{-}\right]=0 \\
& \frac{\mathrm{d}\left[{ }^{-} \mathrm{P}_{\mathbf{i}}\right]}{\mathrm{d} t}=2 k_{\mathrm{D}}\left[{ }^{-} \mathrm{P}_{1}^{-}\right]-k_{11}\left[{ }^{-} \mathrm{P}_{\mathrm{i}}\right]\left(\left[{ }^{-} \mathrm{P}_{\mathrm{i}}\right]+2\left[{ }^{\cdot} \mathrm{P}_{\mathbf{i}}\right]+\left[\mathrm{P}_{\mathbf{i}}\right]\right) \\
& -k_{1}\left[{ }^{-} \mathrm{P}_{\mathrm{i}}\right]-k_{\mathrm{D}}\left[{ }^{-} \mathrm{P}_{\mathrm{i}}\right]=0 \\
& \frac{\mathrm{d}\left[{ }^{\cdot} \mathrm{P}_{\mathrm{i}}\right]}{\mathrm{d} t}=k_{\mathrm{D}}\left[{ }^{-} \mathrm{P}_{\mathrm{i}}\right]-2 k_{11}\left[\mathrm{P}_{\mathrm{i}}\right]\left(\left[{ }^{-} \mathrm{P}_{\mathrm{i}}\right]+2\left[\mathrm{P}_{\mathrm{i}}\right]+\left[\mathrm{P}_{\mathrm{i}}\right]\right) \\
& -2 k_{1}\left[\mathrm{P}_{\mathrm{i}}\right]=0 \\
& \frac{\mathrm{d}\left[\mathrm{P}_{1}^{-}\right]}{\mathrm{d} t}=k_{1}\left[{ }^{-} \mathrm{P}_{\mathrm{i}}\right]-k_{\mathrm{D}}\left[\mathrm{P}_{1}^{-}\right]=0 \\
& \frac{\mathrm{d}\left[\mathrm{P}_{\mathrm{i}}\right]}{\mathrm{d} t}=k_{\mathrm{D}}\left[\mathrm{P}_{1}^{-}\right]+2 k_{1}\left[\mathrm{P}_{\mathrm{i}}\right] \\
& -k_{11}\left[\mathrm{P}_{i}\right]\left(\left[{ }^{-} \mathbf{P}_{i}\right]+2\left[{ }^{\cdot} \mathbf{P}_{i}\right]+\left[\mathrm{P}_{i}\right]\right) \\
& -k_{1}\left[\mathrm{P}_{i}\right]=0
\end{aligned}
$$

where $v, V, t$, and $C$ are the volume of living polymer solution, the volume of deactivating solution which contains TPMB and HPE, time, and the concentration of living polymer (see Figure 1). 
In this series of equations, one can approximate as follows, when $\left[\mathbf{P}_{1}\right] \gg\left[\mathbf{P}_{2}\right],\left[\mathbf{P}_{3}\right],\left[\mathbf{P}_{4}\right]$, or, in other words, when the reaction $\left(\mathbf{P}_{1} \rightarrow \mathrm{P}_{1}\right)$ is very rapid and the reactions $\left(\mathrm{P}_{1} \rightarrow \mathbf{P}_{2}, \mathbf{P}_{3}, \mathbf{P}_{4}\right)$ are very slow.

$$
k_{11}\left(\left[{ }^{-} \mathrm{P}_{\mathrm{i}}\right]+2\left[\mathrm{P}_{\mathrm{i}}\right]+\left[\mathrm{P}_{\mathrm{i}}\right]\right)+k_{1}+k_{\mathrm{D}} \simeq k_{1}+k_{\mathrm{D}}
$$

Then,

$$
\begin{aligned}
& \frac{\mathrm{d} u}{\mathrm{~d} t} \frac{C}{V}=\gamma \\
& {\left[-\mathrm{P}_{1}^{-}\right] \simeq \frac{\gamma}{2 k_{\mathrm{D}}}, \quad\left[{ }^{-} \mathrm{P}_{\mathrm{i}}\right] \simeq \frac{\gamma}{\left(k_{1}+k_{\mathrm{D}}\right)}} \\
& {\left[\mathrm{P}_{\mathrm{i}}\right] \simeq \frac{k_{\mathrm{D}} \gamma}{2 k_{1}\left(k_{1}+k_{\mathrm{D}}\right)}, \quad\left[\mathrm{P}_{1}^{-}\right] \simeq \frac{k_{1} \gamma}{k_{\mathrm{D}}\left(k_{1}+k_{\mathrm{D}}\right)},} \\
& {\left[\mathrm{P}_{\mathrm{i}}\right] \simeq \frac{\gamma}{k_{1}}}
\end{aligned}
$$

Moreover, $k_{\mathrm{D}}$ is considered to be in the order of $10^{0}-10^{-1}$ ( $k_{\mathrm{T}}$ in eq 1 is considered to be in the order of $10^{1}-10^{2},{ }^{*}$ then considering the experimental condition of this study, $k_{\mathrm{D}}=$ $k_{\mathrm{T}}$ [TPMB] is in this order) while $k_{1}$ is considered to be in the order of $10^{1}-10^{2}$. $^{* *}$ So we can approximate as:

$$
\begin{aligned}
{\left[{ }^{-} \mathrm{P}_{1}^{-}\right] } & \simeq \frac{\gamma}{2 k_{\mathrm{D}}}, \quad\left[{ }^{-} \mathrm{P}_{\mathrm{i}}\right] \simeq \frac{\gamma}{k_{1}}, \\
{\left[\mathrm{P}_{1}\right] } & \simeq \frac{k_{\mathrm{D} \gamma}}{2 k_{1}{ }^{2}}, \quad\left[\mathrm{P}_{1}^{-}\right] \simeq \frac{\gamma}{k_{\mathrm{D}}}, \quad\left[\mathrm{P}_{\mathrm{i}}\right] \simeq \frac{\gamma}{k_{1}}
\end{aligned}
$$

For $j=2$, we have

$$
\begin{aligned}
\frac{\mathrm{d}\left[{ }^{-} \mathrm{P}_{2}^{-}\right]}{\mathrm{d} t}= & k_{11}\left[{ }^{-} \mathrm{P}_{\mathrm{i}}\right]\left[{ }^{-} \mathrm{P}_{1}\right]-2 k_{\mathrm{D}}\left[{ }^{-} \mathrm{P}_{2}^{-}\right]=0 \\
\frac{\mathrm{d}\left[{ }^{-} \mathrm{P}_{2}\right]}{\mathrm{d} t}= & 2 k_{11}\left[{ }^{-} \mathrm{P}_{1}\right]\left[{ }^{-} \mathrm{P}_{i}\right]+2 k_{\mathrm{D}}\left[{ }^{-} \mathrm{P}_{2}^{-}\right]-k_{\mathrm{D}}\left[{ }^{-} \mathrm{P}_{2}\right] \\
& -k_{1}\left[{ }^{-} \mathrm{P}_{2}\right]=0 \\
\frac{\mathrm{d}\left[{ }^{\cdot} \mathrm{P}_{2}\right]}{\mathrm{d} t}= & 4 k_{11}\left[{ }^{\cdot} \mathrm{P}_{1}\right]\left[{ }^{\cdot} \mathrm{P}_{1}^{\cdot}\right]+k_{\mathrm{D}}\left[{ }^{-} \mathrm{P}_{2}^{\cdot}\right]-2 k_{1}\left[{ }^{\cdot} \mathrm{P}_{2}^{\cdot}\right]=0
\end{aligned}
$$

* The rate constants of the reactions of living polymer with many halogenated compounds, measured in our laboratory are in this order of magnitude. These results will be reported in the near future.

** Considering the orders of magnitude of $k_{i j} / k_{1}{ }^{2}$ obtained in this study and the well-known results that $k_{i j}$ is in the order of $10^{7}, k_{1}$ is supposed to be in the order of $10^{1}-10^{2}$.

$$
\begin{aligned}
\frac{\mathrm{d}\left[\mathrm{P}_{2}^{-}\right]}{\mathrm{d} t}= & k_{11}\left[{ }^{-} \mathrm{P}_{i}\right]\left[\mathrm{P}_{i}\right]+k_{1}\left[-\mathrm{P}_{2}^{-}\right]-k_{\mathrm{D}}\left[\mathrm{P}_{2}^{-}\right]=0 \\
\frac{\mathrm{d}\left[\mathrm{P}_{2}^{-}\right]}{\mathrm{d} t}= & 2 k_{11}\left[\mathrm{P}_{1}\right]\left[\mathrm{P}_{i}\right]+2 k_{1}\left[\mathrm{P}_{2}^{\prime}\right]+k_{\mathrm{D}}\left[\mathrm{P}_{2}^{-}\right] \\
& -k_{1}\left[\mathrm{P}_{2}^{-}\right]=0
\end{aligned}
$$

Then

$$
\begin{aligned}
{\left[{ }^{-} \mathrm{P}_{2}^{-}\right] } & \simeq \frac{k_{11} \gamma^{2}}{2 k_{\mathrm{D}} k_{1}{ }^{2}}, \quad\left[{ }^{-} \mathrm{P}_{2}\right] \\
{\left[{ }^{\cdot} \mathrm{P}_{2}\right] } & \simeq \frac{k_{11} k_{\mathrm{D}} \gamma^{2}}{2 k_{1}{ }^{4}}, \quad\left[\mathrm{P}_{2}^{-}\right] \simeq \frac{2 k_{11} \gamma^{2} \gamma^{2}}{k_{1}{ }^{3} k_{1}{ }^{2}}, \quad\left[\mathrm{P}_{2}\right] \simeq \frac{2 k_{11} \gamma^{2}}{k_{1}{ }^{3}}
\end{aligned}
$$

For $j=3$, the concentrations of $\left[{ }^{-} \mathrm{P}_{3}^{-}\right],\left[{ }^{-} \mathrm{P}_{3}\right]$, $\left[\mathbf{P}_{3}\right]$ are considered to be almost nil, because if these values were significant, the concentration of $\left[{ }^{\cdot} P_{4}\right]$ should not be negligible. In such a case, the amounts of $P_{5}$ and $P_{6}$ must be observed as measurable values. In this study, $P_{5}$ and $P_{6}$ are not observed, and we may conclude that the concentrations of $\left[{ }^{-} \mathbf{P}_{3}^{-}\right],\left[{ }^{-} \mathbf{P}_{3}\right]$, and $\left[{ }^{-} \mathbf{P}_{3}\right]$ are nil. Then the kinetic expressions are:

$$
\begin{aligned}
\frac{\mathrm{d}\left[\mathrm{P}_{3}^{-}\right]}{\mathrm{d} t}= & k_{12}\left[\mathrm{P}_{1}\right]\left[{ }^{-} \mathrm{P}_{2}\right]+k_{12}\left[{ }^{-} \mathrm{P}_{2}\right]\left[\mathrm{P}_{2}\right]-k_{\mathrm{D}}\left[\mathrm{P}_{3}^{-}\right]=0 \\
\frac{\mathrm{d}\left[\mathrm{P}_{3}\right]}{\mathrm{d} t}= & 2 k_{12}\left[\mathrm{P}_{i}\right]\left[\mathrm{P}_{2}^{\cdot}\right]+2 k_{12}\left[\mathrm{P}_{i}\right]\left[\mathrm{P}_{2}^{\cdot}\right]+k_{\mathrm{D}}\left[\mathrm{P}_{3}^{-}\right] \\
& -k_{1}\left[\mathrm{P}_{3}^{\prime}\right]=0
\end{aligned}
$$

and the results are

$$
\left[\mathrm{P}_{3}^{-}\right] \simeq \frac{3 k_{11} k_{12} \gamma^{3}}{k_{\mathrm{D}} k_{1}{ }^{4}}, \quad\left[\mathrm{P}_{3}\right] \simeq \frac{3 k_{11} k_{12} \gamma^{3}}{k_{1}{ }^{5}}
$$

The quantities of $\mathrm{P}_{1}, \mathrm{P}_{2}, \mathrm{P}_{3}, \mathrm{P}_{4}$ are expressed as follows.

$$
\begin{aligned}
{\left[\mathrm{P}_{1}\right] } & =\int_{0}^{\tau} k_{1}\left[\mathrm{P}_{1}\right] \mathrm{dt}=\gamma \tau \\
{\left[\mathrm{P}_{2}\right] } & =\int_{0}^{\tau}\left(k_{11}\left[\mathrm{P}_{1}\right]^{2}+k_{1}\left[\mathrm{P}_{2}\right]\right) \mathrm{d} t=k_{11} \frac{\gamma^{2}}{k_{1}{ }^{2}}+2 k_{11} \frac{\gamma^{2}}{k_{1}{ }^{2}} \tau \\
& =\gamma^{2} \frac{3 k_{11}}{k_{1}{ }^{2}} \tau=\left[\mathrm{P}_{1}\right] \frac{3 k_{11}}{k_{1}{ }^{2} \gamma} \\
{\left[\mathrm{P}_{3}\right] } & =\int_{0}^{\tau}\left(k_{12}\left[\mathrm{P}_{1}\right]\left[\mathrm{P}_{2}\right]+k_{1}\left[\mathrm{P}_{3}\right]\right) \mathrm{d} t \\
& =k_{\mathrm{D}} \frac{\gamma}{k_{1}} \frac{2 k_{11} \gamma^{2}}{k_{1}{ }^{3}}+\frac{3 k_{11} k_{12} \gamma^{3}}{k_{1}{ }^{4}} \tau \\
& =5 k_{11} k_{12} \frac{\gamma^{3}}{k_{1}{ }^{4}} \tau=\frac{5}{3}\left[\mathrm{P}_{2}\right] \frac{k_{12} \gamma}{k_{1}^{2}} \\
{\left[\mathrm{P}_{4}\right] } & =\int_{0}^{\tau}\left(k_{13}\left[\mathrm{P}_{1}\right]\left[\mathrm{P}_{3}\right]+k_{22}\left[\mathrm{P}_{2}\right]\left[\mathrm{P}_{2}\right]\right) \mathrm{d} t
\end{aligned}
$$


In eq $15-4$, the term $k_{22}\left[\mathrm{P}_{2}\right]\left[\mathrm{P}_{2}\right]$ can be neglected for the reasons discussed in the following paragraph.

Then $\mathrm{P}_{4}$ becomes:

$$
\left[\mathrm{P}_{4}\right]=\frac{3}{5} \frac{\left[\mathrm{P}_{3}\right] \gamma}{k_{1}^{2}} k_{13}
$$

In the above equations, $\tau$ is equal to the time for the solution of the living polymer to flow out, and is a sufficiently long time compared to the life time of the active species. So we can estimate the ratios $k_{i j} / k_{1}^{2}$ from the observed values of $\mathbf{P}_{1}-\mathbf{P}_{4}, \tau$, and $\gamma$.

\section{RESULTS AND DISCUSSION}

The values $k_{11} / k_{1}^{2}, k_{12} / k_{1}^{2}, k_{13} / k_{1}^{2}$ were estimated by eq 15 and 16 and the observed values of $\left[P_{1}\right]-\left[P_{4}\right]$. The results are reported in Tables $I$ and II, and shown in Figures 3 and 4.

In expt no. 9, 10, and 11, we neglected the term $k_{22}\left[\mathrm{P}_{2}\right]\left[\mathrm{P}_{2}\right]$ when we estimated the values of $k_{13} / k_{1}^{2}$, because in Table $\mathrm{I}, k_{11} / k_{1}^{2}$ is not so large at similar conditions of reaction. The error introduced by neglecting the term $k_{22}\left[\mathrm{P}_{2}\right]\left[\mathrm{P}_{2}\right]$ is supposed to the very small.

In Table II, the amounts of $\left[\mathrm{P}_{2}\right],\left[\mathrm{P}_{3}\right]$, and $\left[\mathrm{P}_{4}\right]$ are remarkably increased compared to those in Table I. This may be explained by the high concentration of polystyryl radical caused by the increment of $\gamma$ in expt no. 9, 10, and 11 .

In so far as our results are reliable, the most interesting fact is that, when we consider the values of $k_{1 j} / k_{1}^{2}$, these values increase when the suffix $j$ increase (Figure 4).

Several authors have predicted the dependency of the termination rate constant $\left(k_{i j} / k_{1}^{2}\right)$ on the molecular weight of the polymer. ${ }^{9-14}$ Important results were derived by Ravinowitch, ${ }^{9,10}$ North and Benson, ${ }^{11,13}$ Allen and Patrick, ${ }^{12}$ Ito, ${ }^{16}$ and Horie, ${ }^{17}$ et al.

The tendency of our result seems to agree with the models ${ }^{11,16,17}$ in which the rate determining step in the termination reaction was considered to be the translational diffusion of the center of the mass of a polymer molecule.

The theoretical estimations, such as those related to the segmental diffusion as the rate determining process ${ }^{11}$ or where a pseudocrystal model was considered for the motion of polymer segments, ${ }^{9,10}$ show an inverse dependency on $j$. Furthermore, the observed dependency of $k_{i j} / k_{1}^{2}$ on $j$ is very high compared to the predicted dependency.

On the other hand, some of the results reported already show a rather slight dependency of $k_{i i}$ on $i$, except Schulz. ${ }^{7}$

Another remarkable point of our results is

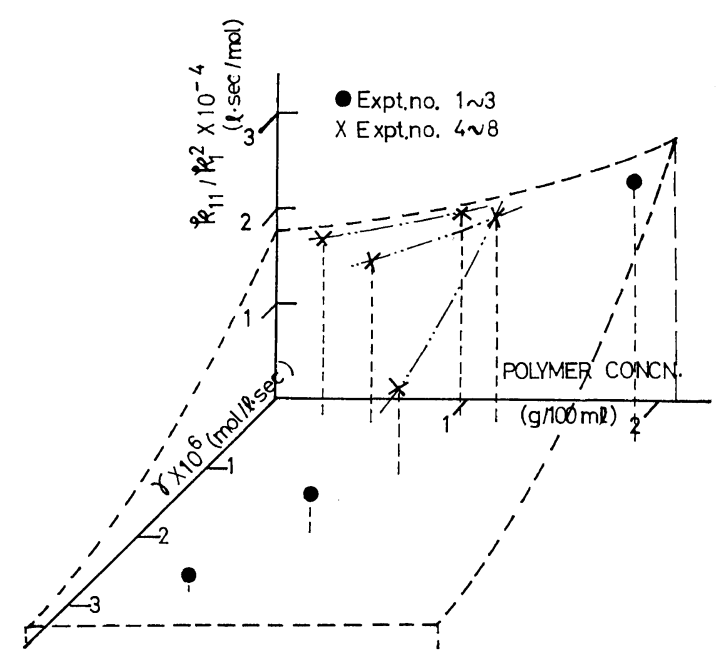

Figure 3. The ratio of the rate constants as a function of polymer concentration and the rate of supply of living polymer solution. (Expt no. 1-8.)

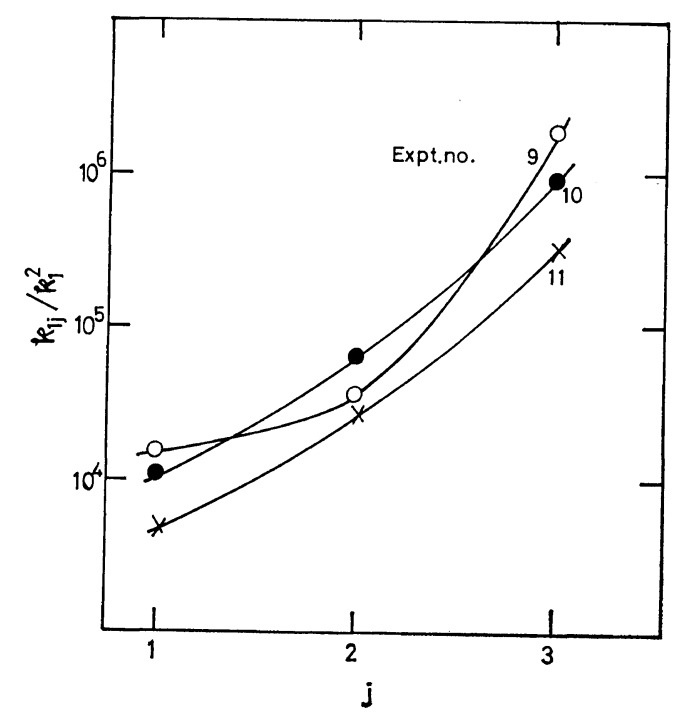

Figure 4. The relationship between the value of $k_{1 j} / k_{1}^{2}$ and $j . \quad$ (Expt no. 9-11.) 
that $k_{11} / k_{1}{ }^{2}$ increases with the increment of polymer concentration. But this apparent result is not so clear when we take into consideration the effect of $\gamma$. (Figure 3). Perhaps it may be valid to consider that there is no difference between each $k_{11} / k_{1}^{2}$ with the polymer concentration within the conditions of this study.

The decrease of $k_{11} / k_{1}{ }^{2}$ with the increment of $\gamma$ may be caused by the rather slow stirring (130 rpm) at part $B$ in Figure 1. This slow stirring caused the local accumulation of triphenylmethyl radical and made $k_{1}$ large.

The influences of these parameters (degree of polymerization, polymer concentration) remain open to further investigations.

To estimate the concentration of each reactive group, we approximate that $k_{11}\left(\left[{ }^{-} \mathrm{P}_{1}\right]+2\left[{ }^{\cdot} \mathrm{P}_{1}\right]+\right.$ [Pi]) and $k_{\mathrm{D}}$ are negligibly small compared to $k_{1}$. The error introduced by this approximation may be minimized if we substitute again the concentration (eq 10) into eq 7 . This treatment may be necessary when $\left[\mathbf{P}_{1}\right]<\left[\mathbf{P}_{2}\right],\left[\mathbf{P}_{3}\right],\left[\mathbf{P}_{4}\right]$, but in the case of this study it seems unnecessary.

As a conclusion, from the tendency of the changes of $k_{1 j} / k_{1}{ }^{2}$ when some factors were changed, the technique proposed in this study may be said to be a new and useful method to observe the value of $k_{1 j} / k_{1}{ }^{2}$ for polymers of definite chain length. The absolute values of $k_{i j}$ and the experimental technique to measure them will be reported in the near future.

\section{REFERENCES}

1. R. G. W. Norrish and R. R. Smith, Nature, (London), 150, 336 (1942).
2. E. Trommsdorff, H. Kohle, and P. Lagally, Makromol. Chem., 1, 169 (1947).

3. A. M. North, "Progress in High Polymer", Vol II, J. C. Robb and F. W. Peaker, Ed., A Heywood Book, London 1968, p 95.

4. K. Yokota, Kobunshi (High Polymers, Japan), 19, No. 220, 566 (1970).

5. J. Hughes and A. M. North, Trans. Faraday Soc., 62, 1886 (1966).

6. A. M. North and Postlewaite, ibid., 62, 2843 (1966).

7. G. W. Schulz and J.P. Fischer, Makromol. Chem., 107, 253 (1967).

8. U. Borgwardt, W. Schnabel, and A. Henglein, ibid., 127, 176 (1969).

9. E. Rabinowitch and W.C. Wood, Trans. Faraday Soc., 32, 1381 (1936).

10. E. Rabinowitch, ibid., 33, 1225 (1937).

11. S. W. Benson and A. M. North, J. Amer. Chem. Soc., 84, 935 (1962).

12. P.E. M. Allen and C. R. Patrick, Makromol. Chem., 72, 106 (1964).

13. A. M. North, ibid., 83, 15 (1965).

14. R. D. Burkhart, J. Polym. Sci., Part A, 3, 883 (1965).

15. Kh. S. Bagdasarian, Vysokomol. Soedin., Ser B, 9, 10 (1967).

16. K. Ito, J. Polym. Sci., Part A-1, 8, 1823 (1970).

17. K. Horie, I. Mita, and H. Kambe, Polymer J., 4, 341 (1973).

18. T. Fujimoto, N. Ozaki, and M. Nagasawa, $J$. Polym. Sci., Part A, 3, 2259 (1965).

19. R. Preckel, and P. W. Selwood, J. Amer. Chem., Soc., 63, 3397 (1941).

20. L. H. Tung, J. Appl. Polym. Sci., 10, 375 (1966).

21. D. H. Richards, N. F. Scilly, and R.L. Williams, Chemical Commn., 322 (1968).

22. R. D. Guthrie and G. R. Weisman, ibid., 1316 (1969). 